\title{
Binding and inhibition in episodic memory-Cognitive, emotional, and neural processes
}

\author{
Karl-Heinz Bäuml *, Bernhard Pastötter, Simon Hanslmayr \\ Department of Experimental Psychology, Regensburg University, 93040 Regensburg, Germany
}

Keywords:

Episodic memory

Retrieval-induced forgetting

Directed forgetting

Think/no-think impaiment

Interference

Competition

Inhibition

Suppression

Affective states

Mood

FMRI

Event-related potentials

Brain oscillations

\begin{abstract}
A B S T R A C T
The goal-directed use of human memory requires that irrelevant or unpleasant memories are, at least temporarily, reduced in their accessibility and memory for more relevant or pleasant information is enhanced, thus making memory more efficient. There is evidence that, in memory, inhibitory processes operate to serve this function. Results from three experimental paradigms are reviewed in which the action of intentionally and unintentionally recruited inhibitory processes has been suggested. The findings provide evidence on representational preconditions for the action of inhibitory processes specifying binding structures in.which inhibitory processes may be triggered and binding structures in which inhibitory processes are generally not observed. The findings also provide evidence on how inhibition affects memory representations, including changes at the memory unit level and changes in the binding between single units. Finally, current knowledge on the interplay between inhibition and emotion and on possible neural correlates of inhibitory processes is reviewed.
\end{abstract}

\section{Contents}

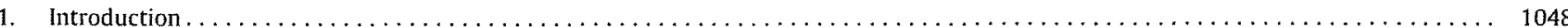

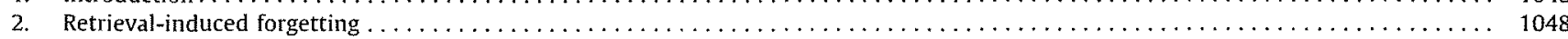

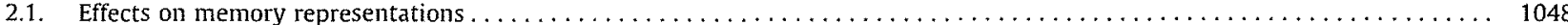

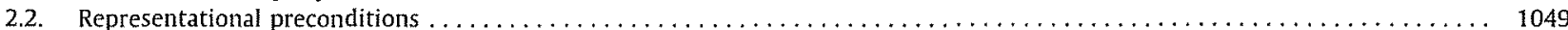

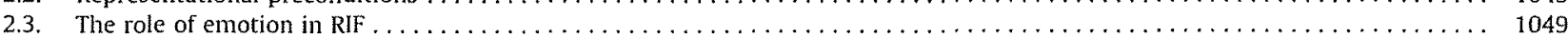

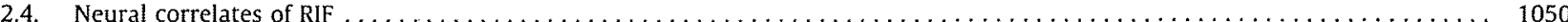

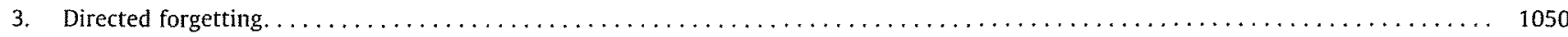

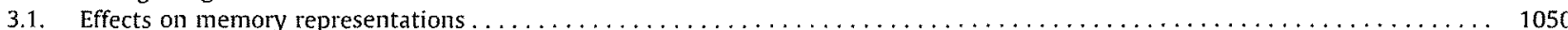

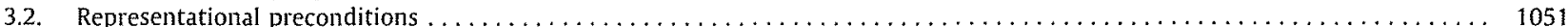

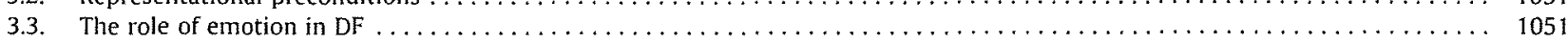

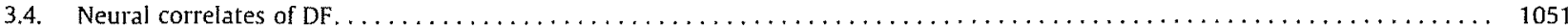

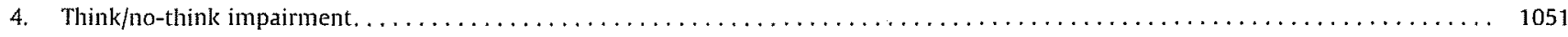

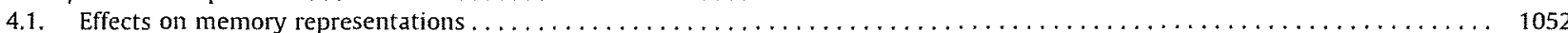

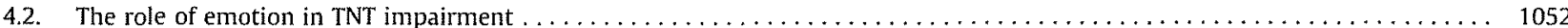

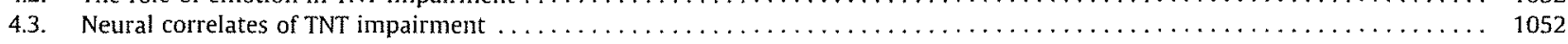

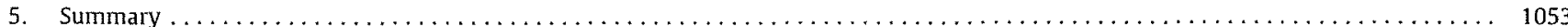

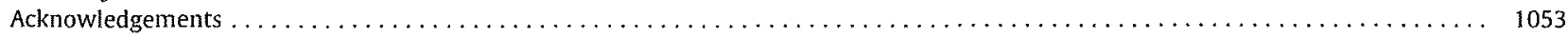

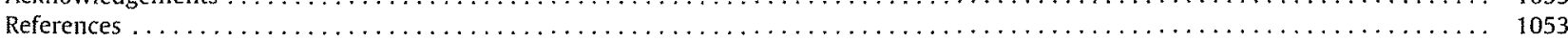

* Corresponding author. Tel.: +49941943 3818; fax: +499419433872.

E-mail address: karl-heinz.baeuml@psychologie.uni-regensburg.de (K.-H. Bäuml). 


\section{Introduction}

A central feature of our episodic memory is retrieval competition. Retrieval competition means that memories bound to a common cue - be it a contextual, semantic, or emotional cue compete for conscious recall when that cue is provided and, as a result, show impaired memory performance (see Crowder, 1976). Retrieval competition provides a challenge for the goal-directed use of memory, because relevant and irrelevant information are often bound to a common cue. This may be the case for the more relevant and more irrelevant news we read in the newspaper, the expired and current passwords of our computer, or the past and current telephone number of a friend. Without effective control mechanisms that attenuate interference from the competing irrelevant material, remembering of the relevant material may fail because the irrelevant information is retrieved.

It has repeatedly been suggested that, in human memory, inhibitory processes operate to serve the function of goal-directed remembering, reducing the binding of competing irrelevant information and enhancing that of the more relevant one. Hereby, mainly two inhibitory mechanisms have recently been entertained to serve the goal of effectively separating relevant from irrelevant information and enhancing memory for the first at the expense of the second: route deactivation and item suppression. Route deactivation means that the binding between the irrelevant information and its cue(s) is weakened, so that retrieval of the irrelevant information becomes less effective (Bjork, 1989). Item suppression means that, rather than "only" weakening a specific retrieval route, inhibition might affect the representation of the irrelevant material itself (Anderson and Spellman, 1995), for instance, by reducing the binding of intra-item features. Due to the direct effect on the memory representation itself, item suppression is supposed to reflect a strong form of inhibition and should be visible over a wide range of memory tests, including, for instance, recognition tasks. In contrast, route deactivation does not affect the representation of the irrelevant material itself, and thus its effects should be restricted to free and cued recall tasks and not be present in recognition tasks. ${ }^{1}$

Inhibitory processes may differ depending on whether they affect the binding of an item's features or the binding between the item and its cue(s). However, inhibitory processes may also differ in whether they are recruited intentionally or unintentionally. In research on unintentional forgetting, for instance, the selective retrieval of previously encoded information has been shown to impair later recall of related material without an explicit intention to forget (Anderson et al., 1994). In contrast, in research on intentional forgetting, it has been found that the presentation of an explicit forget cue can lead to intentional forgetting of previously encoded information (Geiselman et al., 1983). It has even been shown that by simply asking individuals to exclude an unwanted memory from consciousness, later forgetting of the suppressed information can arise (Anderson and Green, 2001). Importantly, in all three cases, the action of inhibitory processes serving memory's goal-directed use has been suggested, indicating a role for both intentionally and unintentionally recruited inhibitory processes in episodic memory.

Here we review results from three experimental paradigms in which a role for inhibitory processes has been suggested: retrievalinduced forgetting, directed forgetting, and think/no-think impair-

\footnotetext{
1 There is some variation in the literature on when exactly a mechanism is called inhibitory. Some researchers speak of inhibition whenever a mechanism reduces accessibility of competing material, be it directly or indirectly (e.g.. Bjork, 1989; Roediger, 1974). Others speak of inhibition only if a mechanism affects the competing material directly (e.g., Anderson, 2003). In the present study, the first view is taken and inhibition is not required to affect the representation of items itself (see also Bäuml, 2008)
}

ment. In retrieval-induced forgetting inhibitory processes are recruited unintentionally, whereas in directed forgetting and think/no-think impairment they are recruited intentionally. For each of the three paradigms we review (1) the basic procedure and suggested inhibitory mechanisms, (2) the observed effects of inhibition on memory of the irrelevant material, (3) the representational preconditions for the action of the inhibitory process, (4) the role of emotion for this type of inhibition, and (5) current knowledge on the neural correlates of the specific form of inhibition. A final summary section will compare results from all three experimental paradigms and offer suggestions on how inhibitory processes vary with the specific empirical situation.

\section{Retrieval-induced forgetting}

Retrieving a subset of formerly studied material can cause subsequent forgetting of the nonretrieved material. Such retrievalinduced forgetting (RIF) has repeatedly been demonstrated using the retrieval-practice paradigm (Anderson et al., 1994; for a review, see Anderson, 2003). In this paradigm, participants study items from different semantic categories (e.g., Fruir-Orange, FruitCherry, InsECr-Bee). Then, in a subsequent practice phase, they repeatedly retrieve half of the items from half of the studied categories using a word stem completion task (e.g., Frurt-Or_) After a distractor task, memory performance for all initially studied items is tested. The typical result in this experiment is that, relative to the control items from the unpracticed categories (Bee), memory for the practiced material (Orange) is improved and memory for the unpracticed material (Cherry) is impaired (see Fig. 1). The two effects of retrieval practice are adaptive to an efficiently functioning memory system, because access to the practiced information, likely to be needed again in the near future, is increased, while interference from competing, currently not needed information, is reduced.

RIF is a retrieval-specific effect (e.g., Anderson et al., 2000a; Bäuml, 2002). While the beneficial effect of retrieval practice mimics the effect of relearning a subset of previously studied items by strengthening the reprocessed material and improving its later memory, only retrieval practice (FrurT-Or_) but not relearning (Frurr-Orange) induces forgetting of the not reprocessed material. RIF is also a very general phenomenon and has been observed with verbal (e.g., Anderson et al., 1994), visual (e.g., Ciranni and Shimamura, 1999), and autobiographical material (Barnier et al., 2004). It has mostly been observed in young adults but is present in young children (Zellner and Bäuml, 2005) and older adults (Aslan et al., 2007) as well.

\subsection{Effects on memory representations}

It is widely assumed that item suppression is the primary mechanism mediating RIF. Following this account, the assumption is that during retrieval practice on a subset of studied material, related unpracticed items interfere and compete for conscious recall. To reduce the interference, the unpracticed material is inhibited, affecting the unpracticed items' memory representation (Anderson and Spellman, 1995), possibly by reducing the binding of intra-item features (for a noninhibitory account, see Williams and Zacks, 2001). Due to the direct effect on the memory representation of the irrelevant information itself, such item suppression should be visible over a wide range of memory tests, including recognition tasks and tasks which employ so-called independent probes, i.e., probes not used until the test phase of an experiment.

Numerous experiments in the past ten years addressed the issue. A large number of these studies demonstrated RIF in free and cued recall tasks (e.g., Anderson et al., 1994; Anderson and 
(A)

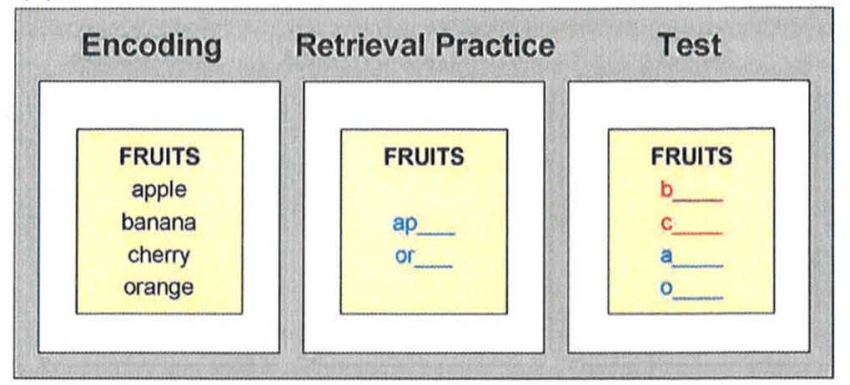

(B)

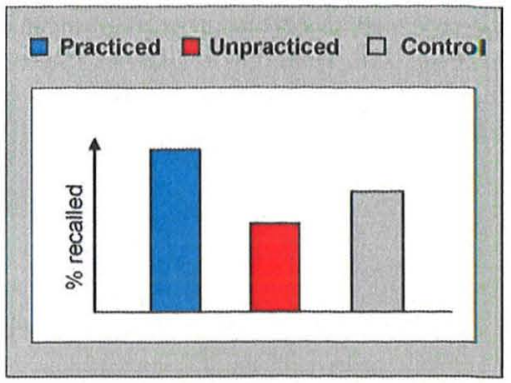

Fig. 1. Retrieval-induced forgetting. (A) Experimental procedure. Participants study a categorized item list. In a subsequent retrieval practice phase, half of the items from half of the studied categories are repeatedly retrieved. On the final memory test, participants are asked to recall all previously studied items. (B) Basic pattern of results. Practiced items show higher recall rates and unpracticed items from practiced categories show lower recall rates than control items from not retrieval-practiced categories.

Spellman, 1995). More important, using the independent-probe procedure, RIF has been reported to be cue independent, i.e., to generalize to cues other than those used at study or retrieval practice (e.g., Anderson and Spellman, 1995; Aslan et al., 2007); for instance, retrieval practice of FrurT-Orange was found to induce forgetting of Banana not only when Banana was tested with the same cue as was used at study and retrieval practice (Frurt), but also when it was probed with a new, independent cue (e.g., MonkEY; for failures to find cue independence, see Camp et al., 2007; Williams and Zacks, 2001).

RIF has also been found in recognition tests (e.g., Hicks and Starns, 2004; Spitzer and Bäuml, 2007; Verde, 2004). Individuals can base their recognition judgments on conscious recollection of information about the spatio-temporal context of a studied episode; however, they can also assess their subjective degree of stimulus familiarity in the absence of any recollective details (e.g., Yonelinas, 2002). Examing recognition memory in RIF, effects on both recollective and familiarity processes of the unpracticed material were reported. While studies in which associative recognition was employed reported a reduction in recollective processes (Verde, 2004), studies in which item recognition was employed reported additional reductions in familiarity processes (Spitzer and Bäuml, 2007).

The results from all these studies are consistent with the view that RIF reflects impairments in the unpracticed items' memory representation itself. This view is further supported by response latency analysis. Examining recall dynamics, Bäuml et al. (2005) found that unpracticed material is reduced in recall level but is not affected in response latency, mimicking typical effects of binding manipulations as they occur as a result of variations in study time or study trials (e.g., Rohrer, 1996). The parallel suggests that retrieval practice affects the unpracticed items' memory representation itself, creating a recovery problem for inhibited items.

\subsection{Representational preconditions}

Retrieval competition is a necessary condition for RIF. Indeed, only when practiced and unpracticed items are bound to the same contextual and/or semantic cue(s), unpracticed items show reduced memory performance as a result of retrieval practice. Thus, if subjects study items from different semantic categories, like Fruit-Orange, FruIT-Cherry, and InSECT-Bee, retrieval practice on Orange will lead to reduced memory performance for Cherry (Anderson et al., 1994), but will not affect performance of Bee; indeed, Bee, being bound to a different semantic cue than Cherry, will show the same performance regardless of whether Orange was previously practiced or not (Shaw et al., 1995).

While retrieval competition is necessary for RIF, the strength of the competition between practiced and unpracticed material seems to play only a minor role for forgetting. In fact, similar levels of forgetting were found when items were practiced which were strongly, moderately, or weakly bound to the common cue, and similar levels of forgetting were found when the unpracticed items were highly or moderately bound to the common cue (Anderson et al., 1994; Bäuml, 1998). Only unpracticed items with a very poor cue-item binding seem to be immune against RIF (Anderson et al., 1994; Bäuml, 1998), suggesting that very low levels of interference may not trigger inhibitory processes when competing material is retrieval practiced.

Following the view that retrieval competition is necessary for RIF, several studies showed that, if retrieval competition is reduced by triggering inter-item binding between the practiced and unpracticed material, forgetting can be eliminated. Consistently, both instructions to interrelate practiced (ANImAL-Tiger) and unpracticed items (ANIMAL-Horse) in a meaningful way (FouR-LEGGED Animal; Anderson et al., 2000b) and the use of strong preexperimental associations between practiced (ANIMAL-CARNIVORETiger) and unpracticed items (ANIMAL-CARNIVORE-Lion; Bäuml and Hartinger, 2002) have been shown to eliminate RIF (for a related result, see Bäuml and Kuhbandner, 2003).

\subsection{The role of emotion in RIF}

Several recent studies addressed the role of emotion in RIF. Doing so, two related, but separate questions arise. The one question is whether RIF differs for emotional and nonemotional contents, independently of people's affective states during retrieval. The other is whether the affective state experienced during retrieval modulates forgetting. The first question has been addressed in three recent studies in which the emotional content of to-be-remembered words, pictures, and self-generated autobiographical memories was varied. The results of the three studies were consistent and suggest that RIF is present with emotional material, and that the amount of forgetting is similar for emotional and nonemotional contents (Amir et al., 2001; Barnier et al., 2004; Kuhbandner et al., in press).

The second question of whether affective states during retrieval affect RIF has been addressed in a recent study by Bäuml and Kuhbandner (2007). In this study, positive, negative, and neutral moods were induced immediately before the retrievalpractice phase. Emotional or nonemotional pictures were presented and participants were told to let the pictures influence their emotional state. Repeated retrieval caused forgetting of unpracticed material when participants were in neutral or positive mood, but did not induce forgetting when participants were in negative mood. Negative mood is known to induce processing of targets by their features and distinctive qualities (e.g., Clore and Huntsinger, 2007). Negative moods thus seem to 
reduce interference from memories that are bound to the same cue and thus reduce inhibition and RIF.

\subsection{Neural correlates of RIF}

Applying fMRI, event-related potentials, and brain oscillations, several recent studies investigated the neural processes underlying RIF. Neural correlates of RIF were examined during the retrieval practice phase and during the final memory test.

Two studies (Johansson et al., 2007; Wimber et al., 2009) compared the neural activity elicited in a retrieval-practice condition (FrulT-Or__ ) with activity in a relearning condition in which selective retrieval did not occur (Frurr-Orange). Johansson et al. (2007) showed that the retrieval condition elicited a stronger positivity over frontal electrode sites compared to the relearning condition. This stronger positivity predicted subsequent forgetting. Similarly, the fMRI study by Wimber et al. (2009) showed that selective retrieval leads to activation of the dorso-lateral prefrontal cortex (DLPFC). Activity in the anterior cingulate cortex (ACC) and the DLPFC was correlated with subsequent forgetting. Another fMRI study (Kuhl et al., 2007) showed that the BOLD signal in the PFC decreases with increasing retrieval practice trials, indicating a decrease of cognitive control demands with repeated selective retrieval. Activity in ventro-lateral (VL) portions of the PFC predicted the subsequent forgetting.

Concerning the effects of retrieval practice on the final memory test, two fMRI studies (Kuhl et al., 2008; Wimber et al., 2008) and one EEG study (Spitzer et al., 2009) have been conducted to date. The fMRI study by Wimber et al. (2008) showed that retrieval of the unpracticed material was related to increased activation in the left anterior region of the VLPFC (BA 47; but see Kuhl et al., 2008). This result can be seen as evidence for the item suppression account of RIF, because increased activation in this brain region (BA 47 ) is implicated to reflect the retrieval of weakly bound memory traces (see Badre and Wagner, 2007). Examining the electrophysiological correlates of RIF on recognition memory, Spitzer et al. (2009) showed that unpracticed items were characterized by reduced early evoked theta power $(4-7 \mathrm{~Hz})$ and reduced gamma power $(60-90 \mathrm{~Hz})$. Both effects suggest reduced memory signals for unpracticed material, which is consistent with the item suppression view of RIF.

The results are consistent with the view that the ACC detects interference from unpracticed material and the DLPFC and VLPFC resolve the conflict by strengthening the practiced memories and inhibiting the unpracticed memories. At test, the inhibited items show reduced early theta and gamma power and increased BOLD signal in the left anterior VLPFC. This finding suggests that, at test, the memory signal of the unpracticed material is impaired, as should be expected if the prior retrieval practice impaired the unpracticed items' binding level.

\section{Directed forgetting}

List-method directed forgetting (DF) is a form of intentional forgetting in which the accessibility of irrelevant out-of-date information is reduced and retrieval of more relevant, newer information is selectively enhanced. In this paradigm, subjects study two lists of items and, after presentation of List 1 , receive a cue to either forget or continue remembering this list before studying List 2 . When later asked to recall all of the previously presented items, including those originally cued to forget, forgetcued subjects typically show impaired recall of List 1 and improved recall of List 2, reflecting the reduced accessibility of irrelevant information and the improved accessibility of relevant information (see Fig. 2; for a review, see MacLeod, 1998).

DF is a fairly general phenomenon and has been observed with verbal (e.g., Geiselman et al., 1983), visual (Basden and Basden, 1996), and autobiographical material (e.g., Barnier et al., 2007). DF has mostly been observed in young adults but is also present in older adults (e.g., Zellner and Bäuml, 2006). Young children typically fail to show successful DF performance (e.g., Harnishfeger and Pope, 1996).

\subsection{Effects on memory representations}

It is often assumed that DF is mediated by route deactivation (e.g., Bjork, 1989). The assumption is that, by inhibiting List-1 items, the forget cue reduces the binding between List- 1 items and their cue(s) and, due to the resulting decrease in the items' interference potential, simultaneously improves access to List 2 (for noninhibitory accounts, see Benjamin, 2007, or Sahakyan and Kelley, 2002). Because the inhibitory account attributes DF to effects on the List-1 items' retrieval routes and not on the items' memory representation itself, List-1 forgetting should be present in recall tasks but should be absent in recognition tasks.

The results of numerous studies demonstrate robust DF effects in free recall tests (e.g., MacLeod, 1998). The effects have been found regardless of whether the two lists were recalled simultaneously in any order the participants wished - i.e., with a tendency to recall List- 2 items before List-1 items -, or whether they were recalled successively with List-1 items recalled prior to List-2 items (Geiselman et al., 1983; Zellner and Bäuml, 2006). This result indicates that DF does not result from output order biases, with List-2 items blocking recall of List-1 items (see Bäuml, 2008).

DF has also been assessed by means of recognition tests. In most of these studies, no effect of the forget cue emerged for either List-1
(A)

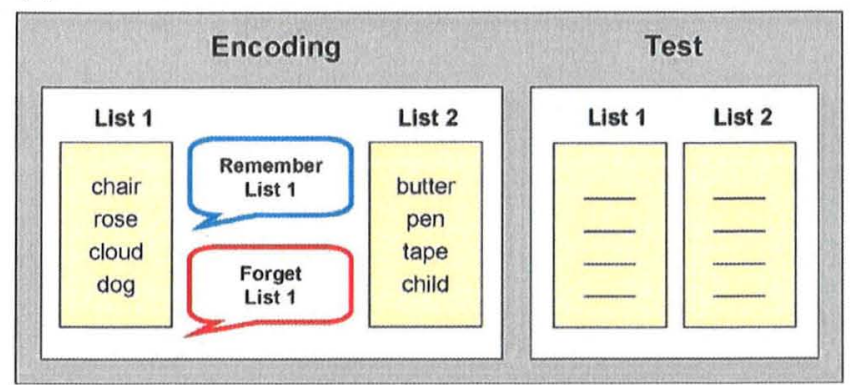

(B)

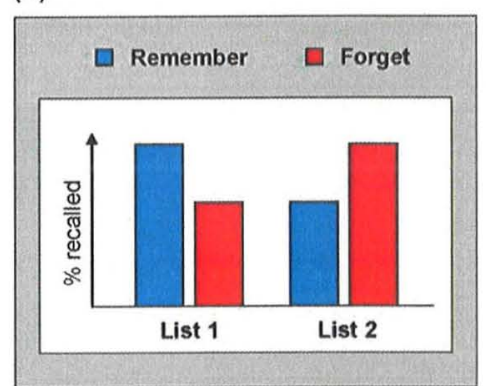

Fig. 2. Directed forgetting. (A) Experimental procedure. Participants study two lists of items and, after the presentation of List 1, receive a cue to either forget or continue remembering this list before studying List 2 . After study of List 2, a recall test is conducted in which participants are asked to recall all previously presented items, including those they were originally cued to forget. (B) Basic pattern of results. Compared to remember-cued participants, forget-cued participants show impaired recall of List-1 and improved recall of List-2 items. 
items or List-2 items (e.g., Basden et al., 1993; Geiselman et al. 1983; but see Benjamin, 2007, for a List-2 effect). Impairments on List-1 recognition, however, were found when participants were required to make source memory judgments (Geiselman et al. 1983), suggesting impaired binding processes after a forget cue is provided. On the basis of the recollection/familiarity distinction, the finding indicates that forgetting in this paradigm reflects primarily a deficit in recollection and not in familiarity.

The results suggest that, in DF, inhibitory processes do not affect the memory representation of the to-be-forgotten items itself but rather affect the binding between List-1 items and their common cue(s). In particular, inhibition in DF seems to operate on a list level rather than an item level, as is suggested by the observation that, when participants alternately learn items intentionally and incidentally, incidentally learned List- 1 items share the same fate as intentionally learned List-1 items (Geiselman et al., 1983; see also Kimball and Bjork, 2002). Thus, List-1 items seem to be bound together to form a unit and the presence of the forget cue induces inhibition of the whole unit.

\subsection{Representational preconditions}

List- 1 forgetting requires the encoding of postcue information that gets bound to the common context cue. Following the original Gelfand and Bjork observation (cited in Bjork, 1989), Pastötter and Bäuml (2007) let subjects study an item list and, after study, subjects received a remember cue or a forget cue. In both conditions, half of the subjects learned a second list, the other half fulfilled an unrelated distractor task. The forget cue induced forgetting of the first list only when participants encoded a second list. The mechanisms responsible for the DF effects thus may operate during List-2 encoding.

Not only the binding of postcue information per se, but also the binding level of the postcue information seems to affect List-1 forgetting. Conway et al. (2000) reduced the binding level of the postcue information by dividing participants' attention during List-2 encoding by means of a secondary task. They found reduced List-1 forgetting in the presence of the secondary task, arguing that strongly bound List-2 items may trigger higher levels of inhibition than weakly bound List- 2 items. Whether List- 1 items with a higher binding level differ in their forgetting from List- 1 items with a lower binding level, however, seems to depend on the binding manipulation. When the binding level of List- 1 items was varied via spaced presentations, strongly bound List-1 items showed more forgetting than weakly bound List- 1 items; variations in processing time and processing depth, however, did not affect forgetting (Sahakyan et al., 2008).

While the results on the role of binding level of List-1 and List-2 items in DF differ from those observed in RIF, the results on the role of inter-item binding in DF are roughly consistent with those for RIF. In RIF, retrieval competition and inhibition have been shown to be reduced when inter-item binding between the practiced and unpracticed material was triggered. Similarly, in DF, List-1 forgetting has been found to be eliminated if List-1 items have bidirectionally related items on List 2 (Conway et al., 2000; Sahakyan and Goodmon, 2007). Obviously, inter-list binding has a strong influence on whether the presence of a forget cue leads to List-1 forgetting or not.

\subsection{The role of emotion in $D F$}

Several recent studies examined the role of emotion in DF. Two studies, employing word lists (Wessel and Merckelbach, 2006) and autobiographical memories (Barnier et al., 2007) as stimuli, addressed the question of whether DF differs for emotional and nonemotional contents. The findings were consistent and suggest that emotional and nonemotional contents do not differ in List-1 forgetting.

The question of whether affective states influence DF has been addressed by Bäuml and Kuhbandner (2009). Assuming that inhibition in DF operates during List-2 encoding (e.g., Pastötter and Bäuml, 2007), the authors examined how affective states modulate DF by inducing positive, negative, and neutral moods immediately before List- 2 encoding. The forget cue caused List- 1 forgetting when participants were in neutral or negative mood, but did not cause forgetting when participants were in positive mood. The absence of DF in positive mood is consistent with the view that positive, but not negative, moods trigger spreading activation processes (e.g., Clore and Huntsinger, 2007). Such processes may lead to reactivation and rebinding of List-1 items during List-2 encoding, thus eliminating the DF effect.

\subsection{Neural correlates of $D F$}

To date, only one study has been reported investigating the neural mechanisms underlying DF (Bäuml et al., 2008). Measuring EEGs during List-2 encoding and analyzing subjects' oscillatory brain activity, Bäuml et al. found that the forget cue induces two effects, an increase in power and a decrease in phase coupling, both observed in the upper alpha frequency band $(11-13 \mathrm{~Hz}$ ); whereas the increase in alpha power predicted List-2 enhancement, the decrease in alpha phase coupling predicted List-1 forgetting. Because phase coupling between electrode sites is regarded a measure of the synchrony between distant neural assemblies (e.g., Lachaux et al., 1999) and coherent firing between distant neuronal populations has been regarded a mechanism subserving binding processes (e.g., Miltner et al., 1999), the decrease in alpha phase coupling could reflect the unbinding of List-1 items and the deactivation of the retrieval routes to List- 1 items. Because results from recent studies suggest that alpha oscillations serve as an active inhibitory filter for the brain (e.g., Klimesch et al., 2007), the finding of the effect in the alpha frequency range supports the view that $\mathrm{DF}$ is mediated by inhibition.

A paradigm formally similar to the DF paradigm is the contextchange paradigm, in which subjects are cued to change their mental context between the encoding of two lists (Sahakyan and Kelley, 2002). On a behavioral level, results from the contextchange paradigm and the DF paradigm are often indistinguishable, consistent with Sahakyan and Kelly's proposal that the DF effects reflect changes in subjects' mental context. Examining oscillatory brain activity in the context-change paradigm, however, it was recently found that oscillatory activities in the context-change paradigm differ qualitatively from those in the DF paradigm (Pastötter et al., 2008), indicating that the DF effects do not reflect internal context change. Together with the Bäuml et al. (2008) study, this study supports the view that DF is caused by inhibition, which reduces the binding between List- 1 items and their cue $(s){ }^{2}$

\section{Think/no-think impairment}

The think/no-think (TNT) paradigm is a memory adaptation of the go/no-go task, which is typically used to study control of prepotent motor responses. In the TNT paradigm, participants study word pairs (e.g., dog-butter) and are trained to answer with the appropriate associate (target) upon presentation of its

\footnotetext{
${ }^{2}$ Directed forgetting has not only been studied using the list method but has also been studied using the so-called item method. The item method differs from the list method in that subjects are cued after each single item whether it should be remembered or should be forgotten. Because item-method directed forgetting, unlike list-method directed forgetting, is mostly attributed to noninhibitory processes (see MacLeod, 1998), the studies addressing neural correlates of itemmethod directed forgetting (e.g., Ullsperger et al., 2000) are not reviewed here.
} 

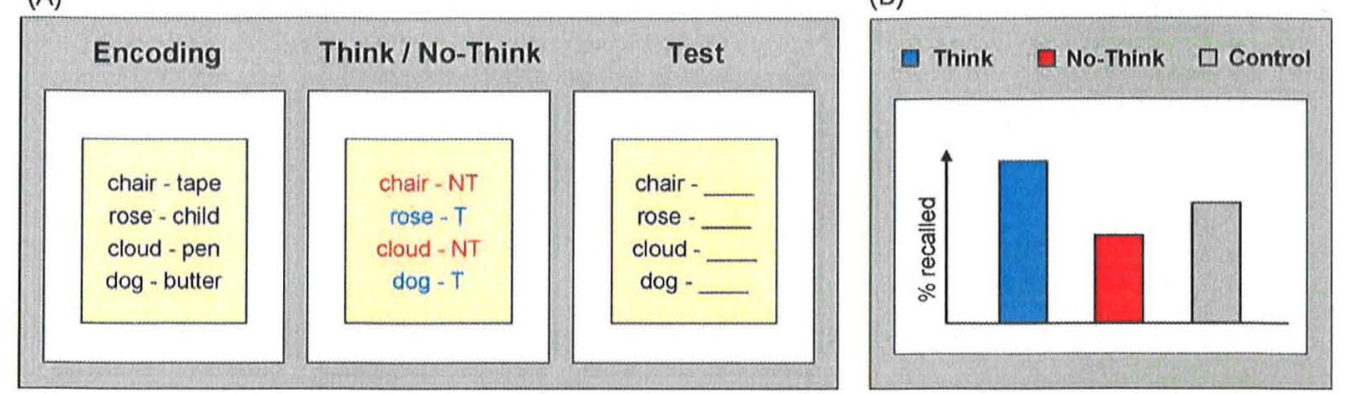

Fig. 3. Think/no-think impairment. (A) Experimental procedure. Participants study cue-target pairs and are trained to answer with the target upon presentation of its cue. After the training, participants engage in the think[T]/no-think[NT] task. In each trial of the task, they are provided with a cue word and are required to either remember (T) or actively suppress any thought (NT) about its corresponding target. On a later memory test, all targets are tested given their associate as retrieval cue. (B) Basic pattern of results. Titems show higher recall rates and NT items show lower recall rates relative to control items that were neither remembered nor suppressed in the intermediate TNT phase.

counterpart (cue). After the training, participants engage in a TNT task. In each trial of the task, the cue word $(\log )$ is provided and participants are required to either remember (think) or actively suppress any thought (no think) about its corresponding target (butter). On a later cued recall test on the target items, subjects typically show improved recall of T items and impaired recall of NT items, relative to baseline items which were neither remembered nor suppressed in the intermediate TNT phase (Anderson and Green, 2001; see Fig. 3).

TNT impairment has been observed with both verbal (e.g. Anderson and Green, 2001; Anderson et al., 2004) and visual material (Depue et al., 2006; Hanslmayr et al., 2009). The results of these studies suggest that typically the forgetting in this paradigm arises if the number of TNT trials is fairly high ( $>10$ trials) but is not present if the number of such trials is relatively low ( $<5$ trials). However, even with a high number of NT trials, the effects are typically small. Correspondingly, some researchers also failed to replicate the finding (e.g., Bergström et al., 2007; Bulevich et al., 2006).

\subsection{Effects on memory representations}

Anderson and Green (2001) argued that the forgetting in the TNT paradigm is caused by item suppression (for a noninhibitory account, see Bulevich et al,, 2006). According to this account, during NT trials, the memory representation of the targets is impaired, possibly by reducing the binding of intra-item features. As a result, accessibility of the items is lowered regardless of which cue is provided and which retrieval route is used. Thus, like RIF, the forgetting should be observable across a wide range of memory tests, including cued recall tasks, independent-probe tasks, or recognition tasks. Several studies addressed the issue reporting TNT impairment in cued recall tests (Depue et al., 2006; Hanslmayr et al., 2009; Hertel and Calcaterra, 2005; but see Bergström et al., 2007, or Mecklinger et al., 2009) and independent-probe tests (Anderson and Green, 2001; Anderson et al., 2004; but see Bulevich et al., 2006). Comparing recognition of NT items with that of T items, Marx et al. (2008) also reported a forgetting effect in a speeded recognition task. No studies have yet been reported examining TNT impairment in other recognition settings.

\subsection{The role of emotion in TNT impairment}

Two studies investigated the role of emotion in TNT impairment, examining whether TNT impairment differs for emotional and neutral material. Depue et al. (2006) reported that item material with negative content induces stronger impairment effects than item material with neutral content. The finding of stronger effects with emotional material was regarded consistent with the view that emotional material is more accessible than nonemotional material (Hamann, 2001) and thus more susceptible to mechanisms of voluntary suppression.

No item material with positive content and no variation of arousal was included in the Depue et al. (2006) study. Therefore, Marx et al. (2008) examined the effects of positive and negative content as well as the effect of arousal in TNT impairment. TNT effects were strongest for both highly arousing items with positive content and lowly arousing items with negative content. These results partly deviate from the results of Depue et al. (2006), possibly because Marx et al. compared recall of NT items directly with recall of T items. Still, the two findings provide first evidence that retrieval of emotional material may be more easily suppressed than neutral material. No studies have yet been reported examining effects of affective states on TNT impairment.

\subsection{Neural correlates of TNT impairment}

So far two fMRI studies examined the neural correlates of TNT impairment (Anderson et al., 2004; Depue et al., 2007). Anderson et al. (2004) showed that suppression trials lead to increased activity of the left and right DLPFC and decreased activity in the left and right hippocampus. Both activations predicted subsequent forgetting. The deactivation in the right hippocampus was correlated with the increased activity in DLPFC, suggesting that DLPFC imposes cognitive control over the hippocampus to keep the unwanted memory from coming to mind.

Using emotional pictures rather than words, Depue et al. (2007) also reported increased activation of PFC regions and decreased activation of the hippocampus during NT trials. Going beyond the prior work, however, Depue et al. found the suppression to be mediated by a two-phase process. They found the early phase of the NT trials to be characterized by increased right VLPFC activation and decreased activation in brain regions related to sensory item processing (e.g., occipital cortex), whereas the later TNT phase was mediated by increased activation of the right middle frontal gyrus and decreased activity in the hippocampus and amygdala. Together, the two studies suggest that suppression in the TNT paradigm is mediated by prefrontal regions which down regulate the activity in memory-related processing areas to keep the unwanted memory from entering consciousness.

Investigating electrophysiological activities during NT suppression, a reduction of sustained positivity was reported to predict forgetting of NT items (Hanslmayr et al., 2009). This effect was evident over right frontal and left parietal electrode sites and appeared to be a reversal of the well-documented cued recall effect, which indicates that stronger sustained positivity is related to successful episodic retrieval (Allan et al., 1996). The results suggest that, during NT trials, retrieval success is voluntarily down 
regulated, inducing later forgetting of the information. In this study, positivity was already reduced in an anticipatory phase, prior to presentation of the memory cue of NT items, suggesting that anticipatory processes prepare the brain for inhibition of the unwanted memory.

Other studies also investigated ERP correlates of TNT impairment (Bergström et al., 2007; Mecklinger et al., 2009). They found a reduction in the left-parietal positivity (LPP) during NT trials, supposedly reflecting the voluntary avoidance of automatic recollection during NT trials. However, because these studies failed to find forgetting of NT items, the functional significance of the LPP reduction for TNT-induced forgetting remains unclear.

\section{Summary}

Different inhibitory mechanisms mediate the modulation in accessibility of irrelevant material in different experimental situations. In RIF, strong evidence exists that retrieval triggers inhibitory processes that affect the memory representation of the irrelevant, not-to-be practiced material directly, possibly by reducing the binding of intra-item features. In TNT, not letting unwanted material enter consciousness also seems to trigger an inhibitory mechanism targeted at the material's memory representation, although here current evidence is limited. In contrast, in DF, strong evidence exists that the forget cue recruits inhibitory processes that do not affect the memory representation of the irrelevant material itself but rather affects the binding between the material and its cue(s). Thus, while in RIF and TNT inhibitory processes induce changes at the memory unit level, in DF inhibitory processes induce changes in the binding between single units.

Although RIF and DF differ in the nature of the underlying inhibitory mechanism, they share the property that forgetting in the two types of situations presupposes that relevant and irrelevant material are bound to a common cue, be it semantic or contextual in nature. This binding structure creates interference between the two types of material, which can be eliminated by enhancing inter-item binding between the relevant and irrelevant material. Correspondingly, several studies showed that both RIF and DF are absent if inter-item binding is enhanced by means of integration processes between the two types of material, indicating that the degree of inter-item binding plays a crucial role for the extent to which inhibitory processes operate in retrieval and in the presence of a forget cue. In contrast, in TNT, relevant and irrelevant material are bound to different cues, so that interference between the two types of material and inter-item binding play no major role for forgetting.

Very recently, several studies examined the possible role of emotion for inhibition. In RIF and DF, both the role of emotional material and the role of affective states were investigated. Although current evidence is limited, the results from these studies suggest that emotional material does not affect RIF and DF, whereas affective states influence both types of forgetting, though in opposing ways. Indeed, whereas negative moods, but not positive moods, seem to eliminate RIF, positive moods, but not negative moods, seem to eliminate DF. Because negative moods induce processing of targets by their distinctive qualities, retrieval of targets in negative mood prevents rebinding of competing material and thus eliminates interference and RIF; in contrast, because positive moods trigger spreading activation processes, they lead to reactivation and rebinding of the irrelevant material, and thus can eliminate DF, although a forget cue was provided. In TNT, no studies examining the role of mood for inhibition have yet been reported. Results from two studies, however, suggest that emotional material may affect TNT impairment, which would contrast with the results for DF and RIF.
Regarding the neural correlates of inhibitory action, fairly detailed knowledge has accumulated on the neural correlates of RIF. The knowledge relies on experiments examining inhibition as it operates, i.e., during the retrieval practice phase, and experiments examining neural markers of inhibition on the final memory test. The knowledge is based on results from fMRI, event-related potentials, and brain oscillations studies and appears well consistent with the view that inhibition in this paradigm affects the memory representation of the irrelevant material itself. Current knowledge on the neural correlates of DF and TNT is restricted to the operating phase of inhibition and does not address possible neural markers of inhibition at test. In TNT, studies using FMRI and event-related potentials have been reported, and the results from both lines of studies are consistent with the view that inhibition in this paradigm is targeted at the material's memory representation. In DF, just a single study using brain oscillatory analysis has been reported. The study provides direct evidence for a reduction in binding processes and supports the view that inhibition in this paradigm reflects a reduction in cue-item binding. However, further work is needed, using a broader range of methods and examining possible neural markers of DF and TNT inhibition on the final memory test.

Together, the reviewed results demonstrate similarities and dissimilarities across the three experimental situations, indicating that different inhibitory processes operate in the different situations. Indeed, dissimilarities show up in the nature of the inhibitory processes, the representational preconditions for inhibition, as well as the interplay between emotion and inhibition. Partly, the dissimilarities may reflect the different degree of intentionality across situations, with RIF reflecting unintentionally recruited inhibition and DF and TNT intentionally recruited inhibition. The dissimilarities, however, may also reflect differences in processing, with inhibition operating during encoding in DF, during selective retrieval in RIF, and during retrieval stopping in TNT.

As a whole, it seems that memory operates very flexibly when trying to reduce the accessibility of irrelevant material, creating mechanisms that are fairly unique for a specific situation. Thus, not a single, general mechanism but a multiplicity of specific memory mechanisms may operate to reduce accessibility of irrelevant material in different situations. Discovering exactly which aspects of a situation determine which of the mechanisms is triggered is a high priority for future research.

\section{Acknowledgments}

The research reported here was supported by a grant from the German Research Foundation (DFG) to Karl-Heinz Bäuml (FOR 448).

\section{References}

Allan, K., Doyle, M.C., Rugg، M.D., 1996. An event-related potential study of wordstem cued recall. Cognitive Brain Res. 4, 251-262.

Amir, N., Coles, M.E., Brigidi, B., Foa, E.B., 2001. The effect of practice on recall of emotional information in individuals with generalized social phobia. J. Abnorm. Psychol. 110, 76-82.

Anderson, M.C. 2003. Rethinking interference theory: executive control and the mechanism of forgetting. J. Mem. Lang. $49,415-445$.

Anderson, M.C., Bjork, R.A., Bjork, E.L., 1994. Remembering can cause forgetting retrieval dynamics in long-term memory. J. Exp. Psychol. Learn. 20, 10631087.

Anderson, M.C., Bjork, E.L., Bjork, R.A., 2000a. Retrieval-induced forgetting: evidence for a recall-specific mechanism. Psychon. B. Rev, 7, 522-530.

Anderson, M.C., Green, C., 2001. Suppressing unwanted memories by executive control. Nature 410, 366-369.

Anderson, M.C., Green, C., McCulloch, K.C., 2000b. Similarity and inhibition in longterm memory: evidence for a two-factor theory. J. Exp. Psychol. Learn. 26, 11411159 . 
Anderson, M.C., Ochsner, K.N., Kuhl, B., Cooper, J., Robertson, E., Gabrieli, S.W. Glover, G.H., Gabrieli, J.D.E., 2004. Neural systems underlying the suppression of unwanted memories. Science 303, 232-235.

Anderson, M.C., Spellman, B.A., 1995. On the status of inhibitory mechanisms in cognition: memory retrieval as a model case. Psychol. Rev, 102, 68-100.

Aslan, A., Bäuml, K.-H., Pastötter, B., 2007. No inhibitory deficit in older adults episodic memory. Psychol. Sci. 18, 72-78.

Badre, D., Wagner, A.D., 2007. Left prefrontal cortex and the cognitive control of memory. Neuropsychologia 45, 2883-2901.

Barnier, A.J., Conway, M.A., Mayoh, L, Speyer, J., Avizmil, O., Harris, C.B., 2007. Directed forgetting of recently recalled autobiographical memories. J. Exp. Psychol. Gen. 136, 301-322.

Barnier, A.J., Hung, L., Conway. M.A., 2004. Retrieval-induced forgetting of emotional and unemotional autobiographical memories. Cognition Emotion 18 457-477.

Basden, B.H., Basden, D.R., 1996. Directed forgetting: further comparisons of the item and list methods. Memory 4, 633-653.

Basden. B.H., Basden, D.R., Gargano, G.J., 1993. Directed forgetting in implicit and explicit memory tests: a comparison of methods. J. Exp. Psychol. Learn. 19, 603616.

Bäuml, K.-H., 1998. Strong items get suppressed, weak items do not: the role of item strength in output interference. Psychon. B. Rev. 5, 459-463.

Bäuml, K.-H., 2002. Semantic generation can cause episodic forgetting. Psychol, Sci, $13,357-361$.

Bäuml, K.-H. 2008. Inhibitory processes In: Roediger, III, HL. (Ed.), Cognitive Psychology of Memory. Learning and Memory: A Comprehensive Reference, vol. 2. Elsevier, Oxford, pp. 195-220.

Bäuml, K.-H., Hansimayr, S., Pastötter, B., Klimesch, W., 2008. Oscillatory correlate of intentional updating in episodic memory. Neurolmage 41, 596-604.

Bäuml, K.-H., Hartinger, A, 2002. On the role of item similarity in retrieval-induced forgetting. Memory 10, 215-224.

Bäuml, K.-H., Kulabandner, C., 2003. Retrieval-induced forgetting and part-list cuing in associatively structured lists. Mem. Cognition 31, 1188-1197.

Bäuml, K.-H., Kuhbandner, $C_{\text {., }}$ 2007. Remembering can cause forgetting-but not in negative moods. Psychol. Sci. 18, 111-115.

BäumI, K.-H., Kuhbandner, C., 2009. Positive moods can eliminate intentional forgetting. Psychon. B. Rev. 16, 93-98.

Bäuml, K.-H., Zellner, M., Vilimek, R., 2005. When remembering causes forgetting: retrieval-induced forgetting as recovery failure. J. Exp. Psychol. Learn, 31, 12211234.

Benjamin, A.S., 2007. The effects of list-method directed forgetting on recognition memory. Psychon. B. Rev. 13, 831-836.

Bergström, Z., Velmans, M., de Fockert, J., Richardson-Klavehn, A., 2007. ERP evidence for successful voluntary avoidance of conscious recollection. Brain Res. 1151,119-133.

Bjork, R.A., 1989. Retrieval inhibition as an adaptive mechanism in human memory. In: Roediger, H.L., Craik, F.I.M. (Eds.), Varieties of Memory and Consciousness: Essays in Honour of Endel Tulving. Lawrence Erlbaum Associates, Hillsdale, pp. 309-330.

Bulevich, J.B., Roediger, H.L., Balota, D.A., Butler III, A.C., 2006. Failures to find suppression of episodic memories in the think/no-think paradigm. Mem. Cognition 34, 1569-1577.

Camp, G., Pecher, D., Schmidt, H.G., 2007. No retrieval-induced forgetting using item-specific independent cues; evidence against a general inhibitory account. J. Exp. Psychol. Learn. 33, 950-958.

Cirami, M.A., Shimamura, A.P., 1999. Retrieval-induced forgetting in episodic memory. 1. Exp. Psychol. Learn. 25, 1403-1414.

Clore, G.L., Huntsinger, J.R., 2007. How emotions inform judgment and regulate thought. Trends Cogn. Sci. 11, 393-399.

Conway, M.A., Harries, K., Noyes, J., Racsmany, M., Frankish, C.R., 2000. The disruption and dissolution of directed forgetting: inhibitory control of memory. $J$ Mem. Lang. 43, 409-430.

Crowder, R.G., 1976. Principles of Learning and Memory. John Wiley \& Sons, New York.

Depue. B.E., Banich, M.T. Curran, T., 2006. Suppression of emotional and nonemotional content in memory. Psychol. Sci. 17, 441-447.

Depue, B.E., Curran, T., Banich, M.T., 2007. Prefrontal regions orchestrate suppression of emotional memories via a two-phase process. Science 317, 215-219.

Geiselman, R.E., Bjork, R.A., Fishman, D., 1983, Disrupted retrieval in directed forgetting: a link with posthypnotic amnesia. J. Exp. Psychol. Gen, 112, 58-72.

Hamann, S., 2001. Cognitive and neural mechanisms of emotional memory. Trends Cogn. Sci. 5, 394-400

Hanslmayr, S., Leipold, P., Pastötter, B., Bäuml, K.-H., 2009. Anticipatory signatures of voluntary memory suppression. J. Neurosci. 29, 2742-2747.

Harnishfeger, KK, Pope, RS, 1996 . Intending to forget: the development of cognitive inhibition in directed forgetting. J. Exp. Child Psychol. 62, 292-315.
Hertel, P.T., Calcaterra, G., 2005. Intentional forgetting benefits from thought substitution. Psychon. B. Rev. 12, 484-489.

Hicks, J.L., Starns, J., 2004. Retrieval-induced forgetting occurs in tests of item recognition. Psychon. B. Rev. 11, 125-130.

Johansson, M., Aslan, A., Bäuml, K.-H., Gäbel, A., Mecklinger, A, 2007, When remembering causes forgetting: electrophysiological correlates of retrieval induced forgetting. Cereb. Cortex 17, 1335-1341.

Kimball, D.R., Bjork, R.A., 2002. Influences of intentional and unintentional forget ting on false memories. J. Exp. Psychol. Gen. 131, 116-130.

Klimesch, W., Sauseng, P., Hanslmayr, S., 2007. Alpha desynchronization revisited: the inhibition/tinjing hypothesis. Brain Res. Rev. 53, 63-88.

Kulbandner, C., Bäuml, K.-H., Stiedl, F.C., in press. Retrieval-induced forgetting of negative stimuli: the role of emotional intensity. Cognition Emotion.

Kuhl, B.A., Dudukovic, N.M., Kahn, 1., Wagner, A.D., 2007. Decreased demands on cognitive control reveal the neural processing benefits of forgetting. Nat Neurosci. 10, 908-914.

Kuhl, B.A., Kahn, 1., Dudukovic, N.M., Wagner, A.D., 2008, Overconing suppression in order to remember: contributions from anterior cingulated and ventrolateral prefrontal cortex. Cogn. Affect. Behav. Neurosci. 8, 211-221.

Lachaux, J.-P., Rodriguez. E., Martinerie, J., Varela, F.J., 1999. Measuring phase synchrony in brain signals. Hum. Brain Mapp. 8, 194-208.

MacLeod, C.M., 1998. Directed forgetting. In: Golding, J.M., MacLeod, C.M. (Eds,) Intentional Forgetting: Interdisciplinary Approaches. Ertbaum, Mahwah, pp. 1 57.

Marx, B.P., Marshall, P.J., Castro, F., 2008. The moderating effects of stimulus valence and arousal on memory suppression. Emotion 8, 199-207.

Mecklinger, A., Parra, M., Waldhauser, G.T., 2009. ERP correlates of intentional forgetting. Brain Research 1255, 132-147.

Miltner, W.H., Braun, C., Arnold, M., Witte, H., Taub, E., 1999. Coherence of gammaband EEG activity as a basis for associative learning. Nature 397, 434436.

Pastötter, B., Bäuml, K.-H., 2007. The crucial role of postcue encoding in directed forgetting and context-dependent forgetting. J. Exp. Psychol. Learn. 33, $977-$ 982

Pastötter, B., Bäuml, K.-H., Hansłmayr, S., 2008. Oscillatory brain activity before and after an internal context change-evidence for a reset of encoding processes. Neurolmage $43,173-181$.

Roediger III. H.L., 1974. Inhibiting effects of recall. Mem. Cognition 2. 261-269.

Rohrer, D., 1996. On the relative and absolute strength of a memory trace. Mem. Cognition 24, 188-201.

Sahakyan, L., Delaney, P.F., Waldum, E.R., 2008. Intentional forgetting is easier after two "shots" than one. J. Exp. Psychol. Learn, 34, 408-414.

Sahakyan, L., Goodmon, L.B., 2007. The influence of directional associations on directed forgetting and interference. J. Exp. Psychol. Learn. 33, 1035-1049.

Sahakyan, L., Kelley, C.M., 2002. A contextual change account of the directed forgetting effect. J. Exp. Psychol. Learn. 28, 1064-1072.

Shaw, J.S., Bjork, R.A., Handal III, A., 1995. Retrieval-induced forgetting in an eyewitness-memory paradigm. Psychon. B. Rev. 2, 249-253.

Spitzer, B., Bäuml, K.-H., 2007. Retrieval-induced forgetting in item recognition: evidence for a reduction in general memory strength. J. Exp. Psychol. Learn. 33 , 863-875.

Spitzer, B., Hanslmayr, S., Opitz, B., Mecklinger, A., Bäuml, K.-H., 2009. Oscillatory correlates of retrieval-induced forgetting in recognition memory. J. Cogn Neurosci. 21, 976-990.

Ullsperger, M., Mecklinger, A., Müller, U., 2000. An electrophysiological test of directed forgetting: the role of retrieval inhibition. J. Cogn. Neurosci. 12, 924940

Verde, M.F. 2004. The retrieval practice effect in associative recognition. Mem. Cognition 32, 1265-1272.

Wessel, I., Merckelbach, H., 2006. Forgetting "murder" is not harder than forgetting "circle": listwise-directed forgetting of emotional words. Cognition Emotion 20 129-137.

Williams, C.C., Zacks, R.T., 2001. Is retrieval-induced forgetting an inhibitory process? Am. J. Psychol. 114, 329-354.

Wimber, M., Bäuml, K.-H., Bergström, Z., Markopoulos، G.. Heinze, H.-j., RichardsonKlavehn, A., 2008. Neural markers of inhibition in human memory retrieval. J Neurosci. 28. 13419-13427.

Wimber, M., Rutschmann, R.M., Greenlee, M.W., Bäuml, K.-H., 2009. Retrieval from episodic memory: neural mechanisms of interference resolution. J. Cogn. Neurosci 21, 538-549.

Yonelinas, A.P., 2002. The nature of recollection and familiarity: a review of 30 years of research. J. Mem. Lang. 46, 441-517.

Zellner, M., Bäuml, K.-H., 2005. Intact retrieval inhibition in children's episodic recall. Mem. Cognition 33, 396-404.

Zellner, M. Bäuml, K.-H., 2006. Inhibitory deficits in older adults-list-method directed forgetting revisited. J. Exp. Psychol. Learn. 32, 290-300. 